\title{
Resveratrol directly targets DDX5 resulting in suppression of the mTORC1 pathway in prostate cancer
}

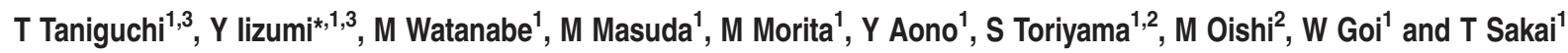

Resveratrol has various attractive bioactivities, such as prevention of cancer, neurodegenerative disorders, and obesity-related diseases. Therefore, identifying its direct binding proteins is expected to discover druggable targets. Sirtuin 1 and phosphodiesterases have so far been found as the direct molecular targets of resveratrol. We herein identified 11 novel resveratrol-binding proteins, including the DEAD (Asp-Glu-Ala-Asp) box helicase 5 (DDX5, also known as p68), using resveratrolimmobilized beads. Treatment with resveratrol induced degradation of DDX5 in prostate cancer cells. Depletion of DDX5 caused apoptosis by inhibiting mammalian target of rapamycin complex 1 (mTORC1) signaling. Moreover, knockdown of DDX5 attenuated the inhibitory activities of resveratrol against mTORC1 signaling and cancer cell growth. These data show that resveratrol directly targets DDX5 and induces cancer cell death by inhibiting the mTORC1 pathway.

Cell Death and Disease (2016) 7, e2211; doi:10.1038/cddis.2016.114; published online 5 May 2016

Resveratrol, a dietary phytochemical enriched in wine, is attracting increasing attention because of its appealing bioactivities, such as prevention of cancer, ${ }^{1,2}$ coronary heart disease, ${ }^{3,4}$ neurodegenerative disorders, ${ }^{5,6}$ and obesityrelated diseases, ${ }^{7,8}$ as well as extending lifespan. ${ }^{9}$ Because of these bioactive potentials, resveratrol has been tested in clinical trials and widely consumed as dietary supplements. ${ }^{10-12}$ To more clearly understand how resveratrol exerts these bioactivities, the direct target molecules of resveratrol have been investigated. ${ }^{13}$ Screening for the activators of sirtuin 1, which was previously considered necessary for the longevity achieved by caloric restriction, ${ }^{14}$ revealed that resveratrol directly activated sirtuin $1 .{ }^{9}$ However, several studies showed that resveratrol indirectly activated sirtuin $1 .^{15,16}$ Resveratrol was subsequently reported to activate sirtuin 1 by directly inhibiting phosphodiesterases (PDEs) ${ }^{17}$ and has recently been suggested again to directly activate sirtuin $1 .{ }^{18}$ Regardless of this controversy, these direct target molecules such as sirtuin 1 and PDEs cannot sufficiently account for other diverse molecular actions of resveratrol. In order to completely comprehend how resveratrol exerts its attractive bioactivities, it is necessary to fully uncover its direct target molecules and clarify the roles of these targets. Furthermore, identifying the direct targets of resveratrol is expected to lead to the discovery of druggable targets. ${ }^{19}$

Resveratrol modulates multiple signaling pathways, for example, by inhibiting the mammalian target of rapamycin complex 1 (mTORC1) pathway. ${ }^{13,20}$ The mTORC1 pathway is known to be deregulated in various human diseases, such as malignant tumors, obesity, type II diabetes, and neurodegenerative diseases. ${ }^{21}$ Especially in malignancies, mTORC1 signaling promotes growth, survival, invasion, metastasis, and angiogenesis, ${ }^{22,23}$ and $\mathrm{mTORC} 1$ inhibitors are used for cancer therapy. ${ }^{21} \mathrm{mTORC} 1$ signaling is regulated by divergent pathways and molecules, such as the phosphatidylinositol 3-kinase pathway, ${ }^{24}$ mitogen-activated protein kinase pathway, ${ }^{25}$ AMP-activated protein kinase (AMPK) pathway, ${ }^{26}$ and astrin. ${ }^{27}$ However, the regulation of the mTORC1 pathway has yet to be clarified and elucidating this will contribute to the development of novel strategies to treat various diseases.

RNA-binding proteins are frequently deregulated in human diseases, such as cancer and neurodegenerative disorders. $^{28,29}$ DEAD (Asp-Glu-Ala-Asp) box helicase 5 (DDX5) is an RNA-binding protein that is overexpressed in various malignant tumors, such as prostate cancer, lung cancer, and ovarian cancer. ${ }^{30}$ The DDX5 gene was shown to be amplified in breast cancer ${ }^{31}$ and fused with ETV4, the E26 transformation-specific transcription factor family gene, in prostate cancer. $^{32}$ DDX5 promotes growth, ${ }^{33}$ metastasis, ${ }^{34}$ and drug resistance ${ }^{35}$ by activating several oncogenic pathways. ${ }^{34,36}$ Although DDX5 also functions as a transcriptional co-activator of the androgen receptor in hormone-dependent prostate cancer, ${ }^{37}$ its roles in hormone-independent prostate cancer have not been clarified.

\footnotetext{
${ }^{1}$ Department of Molecular-Targeting Cancer Prevention, Graduate School of Medical Science, Kyoto Prefectural University of Medicine, Kawaramachi-Hirokoji, Kamigyo-ku, Kyoto 602-8566, Japan and ${ }^{2}$ Department of Urology, Graduate School of Medical Science, Kyoto Prefectural University of Medicine, Kawaramachi-Hirokoji, Kamigyo-ku, Kyoto 602-8566, Japan

*Corresponding author: Y lizumi, Department of Molecular-Targeting Cancer Prevention, Graduate School of Medical Science, Kyoto Prefectural University of Medicine, Kawaramachi-Hirokoji, Kamigyo-ku, Kyoto 602-8566, Japan. Tel: +81 75251 5338; Fax: +81 75241 0792; E-mail: yiizumi@koto.kpu-m.ac.jp

${ }^{3}$ These authors contributed equally to this work.

Abbreviations: DDX5, DEAD (Asp-Glu-Ala-Asp) box helicase 5; mTORC1, mammalian target of rapamycin complex 1; PDE, phosphodiesterase; AMPK, AMP-activated protein kinase; ACC, acetyl-CoA carboxylase; 4EBP1, eukaryotic translation initiation factor 4E-binding protein 1; DMF, N, N-dimethylformamide; TGM4, transglutaminase4; PrLZ, prostate leucine zipper

Received 17.12.15; revised 22.3.16; accepted 23.3.16; Edited by A Stephanou
} 
The present study shows that the inhibition of the mTORC1 pathway and cancer growth by resveratrol is not attributed to the inhibition of PDE. To identify the novel targets of resveratrol, we produced resveratrol-immobilized beads using our previously reported method. ${ }^{38}$ The affinity beads method and proteomic analysis identified 11 novel targets, including DDX5. Resveratrol directly bound to and promoted metalloprotease-dependent degradation of DDX5 protein, leading to cancer cell death with inhibition of the mTORC1 pathway. We demonstrate for the first time that DDX5 is a primary target of resveratrol in tumor suppression.

\section{Results}

Resveratrol, but not the PDE4 inhibitor rolipram, inhibits the $\mathrm{mTORC} 1$ pathway and growth of prostate cancer cells. Resveratrol activates the AMPK pathway and restores aging-related metabolic phenotypes by directly inhibiting PDEs. ${ }^{17}$ To evaluate the roles of PDEs in cancer growth inhibition by resveratrol, we compared the effects of resveratrol with those of the specific PDE4 inhibitor rolipram on the growth of prostate cancer PC-3 cells. As shown in Figure 1a, resveratrol, but not rolipram, inhibited the growth of PC-3 cells. It was reported that AMPK inhibited the mTORC1 pathway, ${ }^{26}$ which promotes the development of prostate cancer, ${ }^{23}$ and also that resveratrol inhibited the mTORC1 pathway. ${ }^{7,39}$ We therefore examined whether these PDE inhibitors, including resveratrol, inhibited the mTORC1 pathway. Although inhibition of PDE increased the phosphorylation of AMPKa at Thr172 and its substrate acetyl-CoA carboxylase (ACC) at Ser79 (Figure 1b), indicating the activation of AMPK, only resveratrol inhibited the phosphorylation of ribosomal protein S6 kinase 1 (S6K1) at Thr389 and eukaryotic translation initiation factor 4E-binding protein 1 (4EBP1), reflecting the activation of mTORC1 (Figure 1c). These results suggest that resveratrol suppresses the mTORC1 pathway and growth of prostate cancer cells independent of the inhibition of PDE.

Identification of DDX5 as a target of resveratrol. As the inhibition of PDE could not suppress the mTORC1 pathway and cancer growth, we speculated the existence of unknown target molecules of resveratrol that were related to its anticancer activity. To identify the novel molecular targets of resveratrol, we generated resveratrol-immobilized beads using our previously described method $^{38}$ (Figure 2a). We first confirmed that purified recombinant PDE4A protein bound to resveratrol-fixed beads (Figure $2 b$ ). Resveratrolbinding proteins were then purified from PC-3 whole-cell extracts using these beads and analyzed by MALDI-TOF MS. The resveratrol-fixed beads method and proteomic analysis uncovered 11 novel resveratrol-binding proteins, including 7 RNA-binding proteins, 2 mitochondrial proteins, 1 transcription factor, and 1 ribosomal protein (Figure 2c and Supplementary Table S1). As one of the resveratrol-binding proteins, the RNA helicase DDX5/p68 was reported to be overexpressed and amplified in several malignant tumors and have oncogenic properties, ${ }^{30,31}$ we focused on the role of DDX5 as a target of resveratrol in cancer inhibition. The competitive assay showed that the binding of DDX5 to resveratrol-fixed beads was competed with free resveratrol,
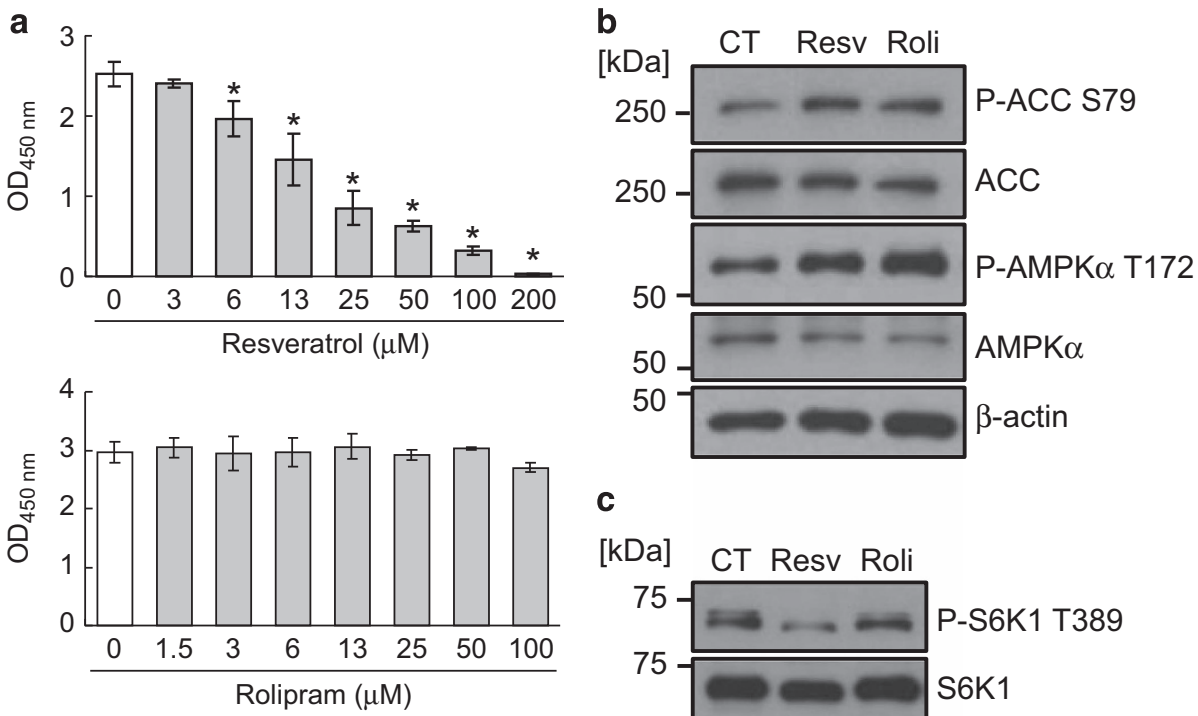

C

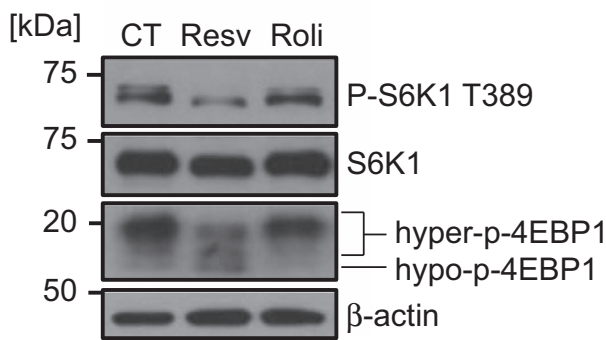

Figure 1 Resveratrol, but not a PDE inhibitor, suppresses the growth of prostate cancer cells. (a) Human prostate cancer PC-3 cells were treated with the indicated concentrations of resveratrol or the PDE4 inhibitor rolipram for $72 \mathrm{~h}$. Relative viability of the cells was measured by CCK-8 assay. Data are means \pm S.D. $(n=3)$. ${ }^{*} P<0.05$ relative to control (one-way analysis of variance, Bonferroni post-hoc tests). (b and c) Western blotting analysis of PC-3 cells treated with $0.1 \%$ DMSO (CT), $100 \mu \mathrm{M}$ resveratrol (Resv), or $100 \mu \mathrm{M}$ rolipram (Roli) for $3 \mathrm{~h}$ (b) or $24 \mathrm{~h}$ (c) 


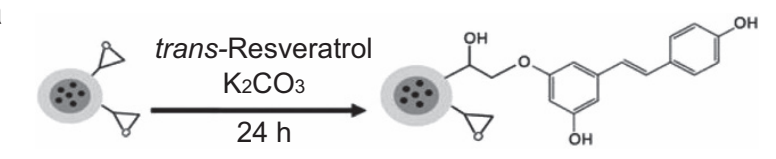

b

[kDa] Input (-) (+)
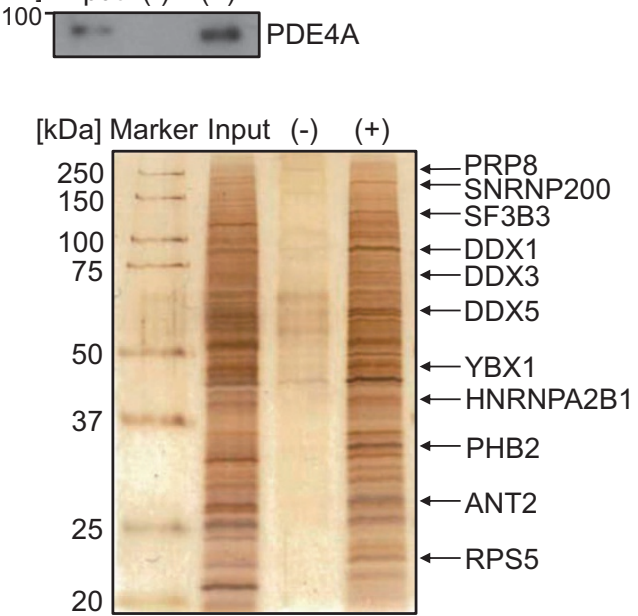

d

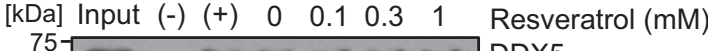
75- - $\rightleftharpoons$ DDX5

e

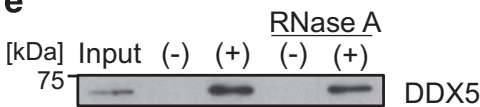

Figure 2 Identification of resveratrol-binding proteins. (a) The scheme for fixation of resveratrol onto magnetic FG beads with epoxy linkers. (b) Purified recombinant PDE4A $(2 \mu \mathrm{g})$ was incubated with empty $(-)$ or resveratrol-immobilized (+) beads for $4 \mathrm{~h}$, and bound PDE4A was detected by western blotting. The input lane corresponds to recombinant PDE4A $(250 \mathrm{ng})$. (c) Resveratrol-binding proteins were purified from human prostate cancer PC-3 cell extracts, silver-stained, and identified by matrix assisted laser desorption/ionization time-of-flight mass spectrometric analysis. The input lane represents $1 \%$ of the PC- 3 cell extracts used for the binding assay. (d) In the competitive assay, PC-3 cell extracts were preincubated with the indicated doses of resveratrol for $1 \mathrm{~h}$ and incubated with the beads for $15 \mathrm{~min}$. Bound DDX5 was detected by western blotting. The input lane represents $5 \%$ of the PC-3 cell extracts used for the binding assay. (e) Purified recombinant DDX5 $(1 \mu \mathrm{g})$ with or without RNase A was incubated with the beads, and bound DDX5 was detected by western blotting. The input lane corresponds to recombinant DDX5 protein (150 ng)

suggesting that resveratrol specifically bound to DDX5 (Figure 2d). Furthermore, purified recombinant DDX5 bound to resveratrol-fixed beads regardless of the digestion of RNA by RNase $A$ treatment (Figure 2e). These findings indicate that resveratrol directly binds to DDX5.

We next evaluated the effects of resveratrol on endogenous DDX5. The endogenous protein levels of DDX5 were dosedependently decreased by the treatment with resveratrol in PC-3 and DU145 cells (Figure $3 a$ and Supplementary Figure S1a). We further investigated whether the physiological concentrations of resveratrol also downregulated DDX5. The once-daily administration of $5 \mathrm{~g}$ of resveratrol to healthy volunteers for 29 days was shown to yield 2.3-7.0 $\mu \mathrm{M}$ of resveratrol in the plasma. ${ }^{40}$ The protein levels of DDX 5 were reduced (Figure $3 b$ ) after treatment with clinically achievable concentrations of resveratrol $(5-10 \mu \mathrm{M})$ every $24 \mathrm{~h}$ for 3 days. These data raise the possibility that DDX5 is a physiological target of resveratrol in the human body.

We further examined the mechanism by which resveratrol downregulated the expression of DDX5 protein. Treatment with resveratrol did not downregulate DDX5 mRNA (Figure 3c). As shown in Figure 3d, DDX5 protein was stable because it was not decreased by the de novo protein synthesis inhibitor cycloheximide, but resveratrol reduced DDX5 protein in the presence of cycloheximide, indicating that resveratrol promoted the degradation of DDX5 protein. Next we tested what types of proteases were related to degradation of DDX5 protein. Only EDTA partially inhibited the degradation of DDX5 by resveratrol, whereas the proteasome inhibitor lactacystin, the autophagy inhibitor bafilomycin A1, and protease inhibitors (leupeptin, antipain, and pepstatin A) did not (Figure 3e). These results suggest that resveratrol degrades DDX5 protein by promoting metalloprotease-dependent degradation.

Depletion of DDX5 expression suppresses the growth of prostate cancer cells by inhibiting the mTORC1 pathway and inducing apoptosis. Although DDX5 is overexpressed in prostate cancer and functions as a co-activator of the androgen receptor, ${ }^{37}$ its functions in hormone-refractory prostate cancer remain unknown. We found that knockdown of DDX5 inhibited the growth and colony formation of hormone-refractory prostate cancer PC-3 and DU145 cells (Figures $4 \mathrm{a}$ and $\mathrm{b}$ ), similar to the treatment with resveratrol (Figure 1a and Supplementary Figure S1b). Knockdown of DDX5 remarkably induced apoptosis in PC-3 cells (Figure 4c), similar to the resveratrol treatment (Supplementary Figure S2). These results suggest that depletion of DDX5 inhibits the growth of hormone-refractory prostate cancer cells with inducing apoptosis.

We then investigated how depletion of DDX5 inhibited the growth of prostate cancer cells. Although depletion of DDX5 was reported to induce apoptosis by downregulating Notch1 signaling in lymphoblastic leukemia cells, ${ }^{36}$ Notch1 signaling was not suppressed by DDX5 depletion in prostate cancer cells (Supplementary Figure S3). We next examined whether knockdown of DDX5 inhibited mTORC1 signaling similar to the treatment with resveratrol (Figure 1c and Supplementary Figure S1a). As shown in Figure 5a, DDX5 knockdown inhibited the mTORC1 pathway in prostate cancer PC-3 and DU145 cells. As dephosphorylation of 4EBPs is critical for causing growth inhibition and apoptosis, ${ }^{41}$ we tested whether knockdown of only 4EBP1, one of the 4EBP family, attenuated the inhibitory effects of DDX5 depletion. The knockdown of 4EBP1 partially inhibited the growth inhibition and apoptosis induced by the depletion of DDX5 (Figures $5 b$ and $c$ and Supplementary Figure S4). These findings indicate that depletion of DDX5 can mimic the inhibitory effects of resveratrol against the $\mathrm{mTORC} 1$ pathway and prostate cancer cell growth.

Resveratrol inhibits the mTORC1 pathway and growth of prostate cancer cells by targeting DDX5. As resveratrol degraded DDX5 protein and the depletion of DDX5 inhibited the mTORC1 pathway and growth of prostate cancer cells, we evaluated the significance of degrading DDX5 in the 
a

\begin{tabular}{|c|c|c|c|c|c|c|c|c|c|c|}
\hline a & & & & Res & verat & ol $(\mu$ & & & & \\
\hline [kDa] & 0 & 3 & 6 & 13 & 25 & 50 & 100 & 0 & 10 & \\
\hline 75 & - & 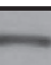 & 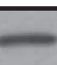 & - & - & - & $\longrightarrow$ & $=$ & 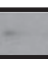 & DDX5 \\
\hline & & & & & & & & & & $\beta$-actin \\
\hline
\end{tabular}

b
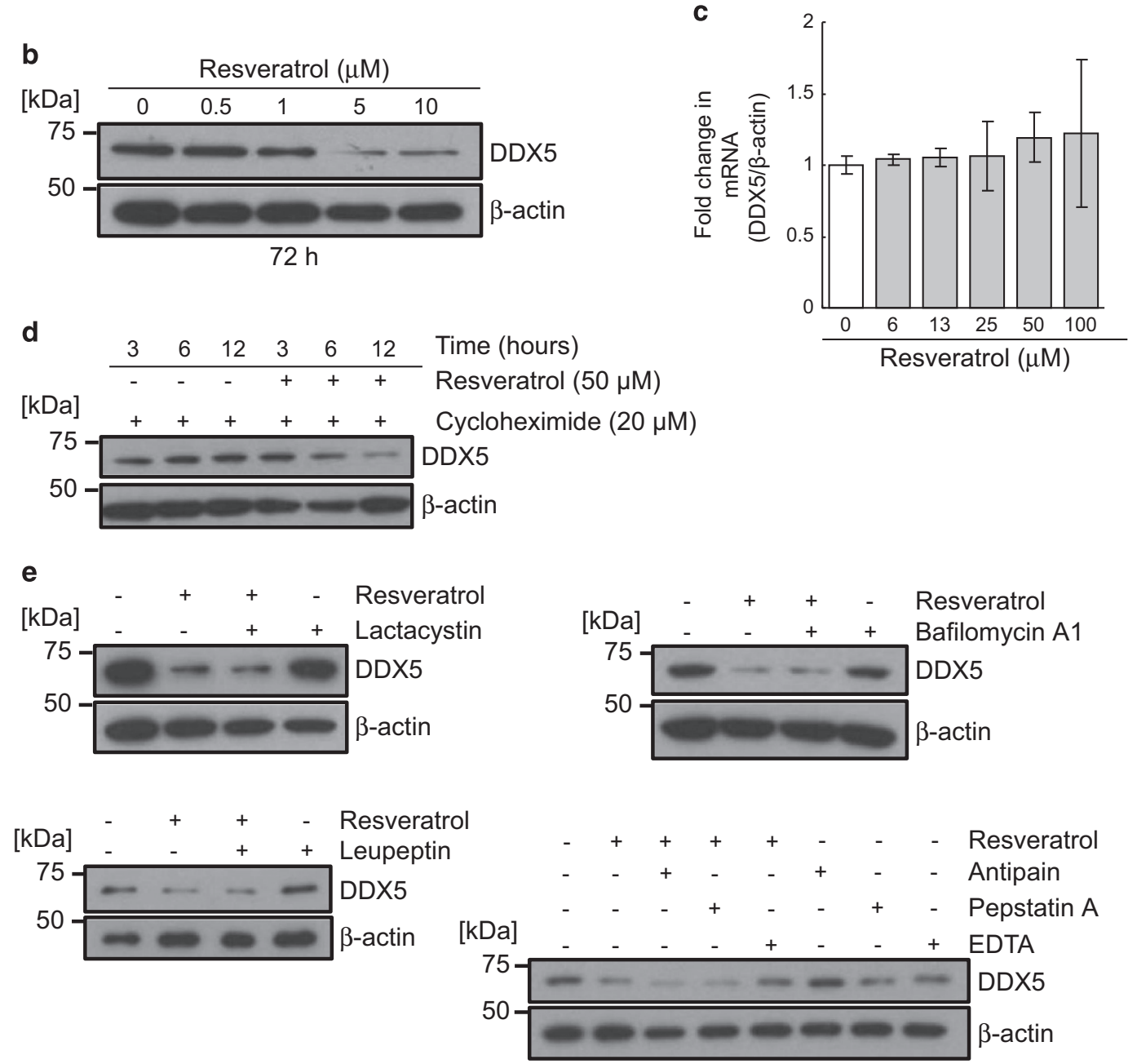

Figure 3 Treatment with resveratrol degrades DDX5 protein. (a and $\mathbf{b})$ PC-3 cells were treated with the indicated concentrations of resveratrol for $24 \mathrm{~h}(\mathbf{a})$ or every $24 \mathrm{~h}$ for $72 \mathrm{~h}$ (b). DDX5 protein was detected by western blotting. (c) Quantitative reverse transcriptase-PCR analysis of DDX5 mRNA in PC-3 cells treated with resveratrol for $24 \mathrm{~h}$. Data are means \pm S.D. $(n=3)$. (d) PC-3 cells were incubated for the indicated times with or without $50 \mu \mathrm{M}$ resveratrol and/or $20 \mu \mathrm{M}$ cycloheximide. DDX5 protein was detected by western blotting. (e) PC-3 cells were incubated for $24 \mathrm{~h}$ with or without $20 \mu \mathrm{M}$ lactacystin, $1 \mu \mathrm{M}$ bafilomycin A1, $100 \mu \mathrm{M}$ leupeptin, $100 \mu \mathrm{M}$ antipain, $1 \mu \mathrm{M}$ pepstatin A, or $10 \mathrm{mM}$ EDTA and/or $50 \mu \mathrm{M}$ resveratrol. DDX5 protein was detected by western blotting

anticancer effects of resveratrol. As shown in Figures $6 a$ and $\mathrm{b}$, the depletion of DDX5 attenuated the growth inhibition and apoptosis caused by resveratrol. Furthermore, the knockdown of DDX5 also attenuated the inhibitory effects of resveratrol on the mTORC1 pathway (Figure 6c). These results suggest that resveratrol inhibits the growth of prostate cancer cells by directly targeting DDX5 (Figure 7).

\section{Discussion}

In the present study, we employed resveratrol-immobilized beads to explore the unknown targets of resveratrol and identified 11 resveratrol-binding proteins. One of the resveratrol-binding proteins, DDX5, was degraded by treatment with physiological concentrations of resveratrol. The depletion of DDX5 protein resulted in growth inhibition and cell death by suppressing mTORC1 signaling in androgenindependent prostate cancer cells, similar to the treatment with resveratrol. Furthermore, these activities of resveratrol were repressed by DDX5 depletion. Taken together, our results suggest that resveratrol suppresses mTORC1 signaling in androgen-independent prostate cancer cells by directly targeting DDX5 (Figure 7), and 10 other resveratrol-binding proteins might also be druggable targets, similar to DDX5. 
a
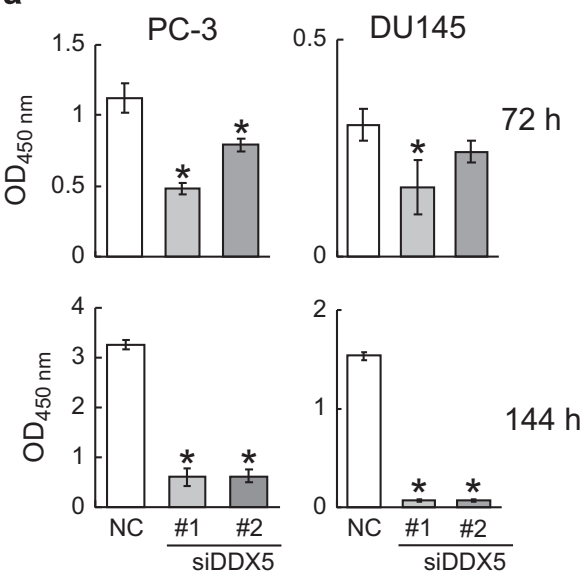

C

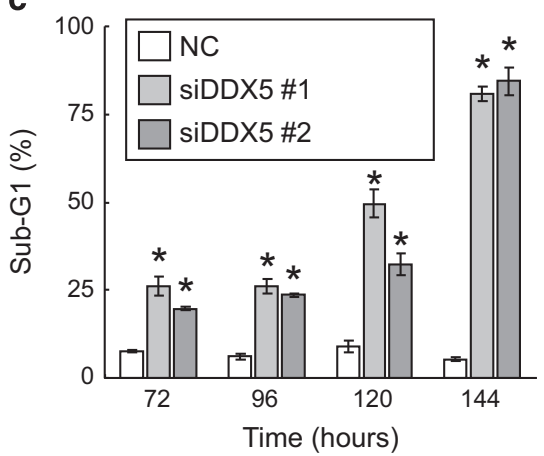

b
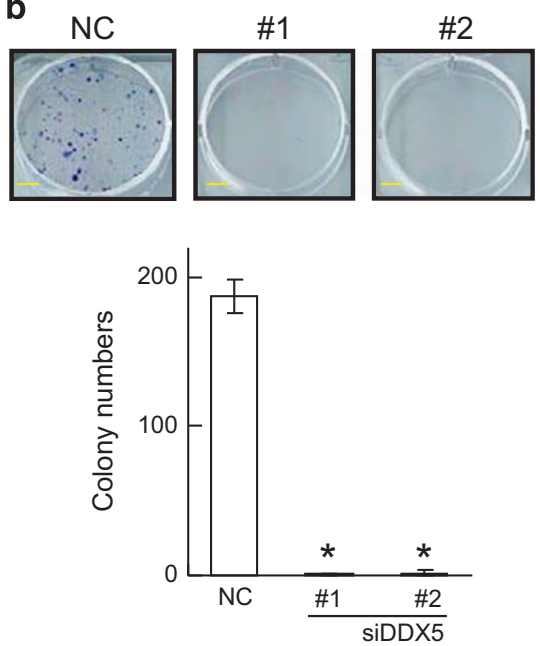

Figure 4 Knockdown of DDX5 inhibits the growth of hormone-independent prostate cancer cells. (a) PC-3 and DU145 cells were transfected with a negative control siRNA (NC), siDDX5 \#1, or siDDX5 \#2 for 72 or $144 \mathrm{~h}$. Relative viability of the cells was measured by CCK-8 assay. Data are means \pm S.D. $(n=3)$. ${ }^{*} P<0.05$ relative to control (one-way analysis of variance (ANOVA), Bonferroni post-hoc tests). (b) After PC-3 cells were transfected with or without siDDX5 and incubated for 9 days, colonies were stained with crystal violet and counted (scale bar: $5 \mathrm{~mm}$ ). Data are means \pm S.D. $(n=3) .{ }^{*} P<0.05$ relative to control (one-way ANOVA, Bonferroni post-hoc tests). (c) PC-3 cells were transfected with or without siDDX5 and incubated for $72,96,120$, or $144 \mathrm{~h}$, and the percentage of sub-G1 population was quantified by flow cytometry. Data are means \pm S.D. $(n=3)$. ${ }^{*} P<0.05$ relative to control (one-way ANOVA, Bonferroni post-hoc tests)

As shown in Supplementary Figure S5, we also found that resveratrol at $100 \mu \mathrm{M}$, but not $50 \mu \mathrm{M}$, downregulated mRNA expressions of transglutaminase-4 (TGM4) and prostate leucine zipper (PrLZ). As TGM4 promotes cellular migration $^{42}$ and PrLZ suppresses apoptosis, ${ }^{43}$ a high concentration of resveratrol might further exhibit other anticancer effects by targeting other resveratrol-binding proteins and downregulating these molecules.

As resveratrol exerts beneficial bioactivities in organisms, its target proteins have been investigated. Although sirtuin 1, one of the most famous targets of resveratrol, was activated by $25 \mu \mathrm{M}$ of resveratrol in cells, ${ }^{18}$ it was difficult for resveratrol to reach $25 \mu \mathrm{M}$ under physiological conditions. ${ }^{40}$ In contrast, it was suggested that resveratrol at $10 \mu \mathrm{M}$ activated the AMPK pathway by directly inhibiting PDEs in cells. ${ }^{17}$ Our study showed that resveratrol degraded DDX5 protein at $5 \mu \mathrm{M}$ every $24 \mathrm{~h}$ for 3 days (Figure $3 \mathrm{~b}$ ). Taken together, DDX5 as well as PDEs may be targeted by resveratrol in organisms, including human beings.

There are currently few efficient agents for castration-resistant prostate $^{\text {cancer. }}{ }^{44}$ In this study, we have suggested that resveratrol inhibits the growth of castration-resistant prostate cancer cells by degrading DDX5. DDX5 is overexpressed and promotes tumor development in prostate and other malignant tumors. ${ }^{30}$ These data raise the possibility that DDX5 is a druggable target for castration-resistant prostate cancer therapy. On the other hand, by screening for p15-inducing compounds, we have discovered the MEK inhibitor trametinib, ${ }^{45}$ which has recently been approved for the treatment of BRAF-mutated melanoma by the US Food and Drug Administration, EU, and others. Screening for DDX5-targeting agents may provide effective drugs for castration-resistant prostate cancer.

At present, docetaxel and antiandrogen agents are mainly utilized in the treatment of castration-resistant prostate cancer. However, as these agents are not sufficient, effective combination therapies are required. As resveratrol and the antiandrogen flutamide synergistically inhibit the androgen receptor, ${ }^{46}$ the combination of resveratrol and flutamide may be a promising therapy. Moreover, it has been reported that the mTORC1 inhibitor rapamycin enhances docetaxel efficacy against the PC-3 xenograft model, a castration-resistant prostate cancer model, by downregulating survivin. ${ }^{47}$ In the present study, resveratrol inhibited the mTORC1 pathway by targeting DDX5, and we raise the possibility that resveratrol 

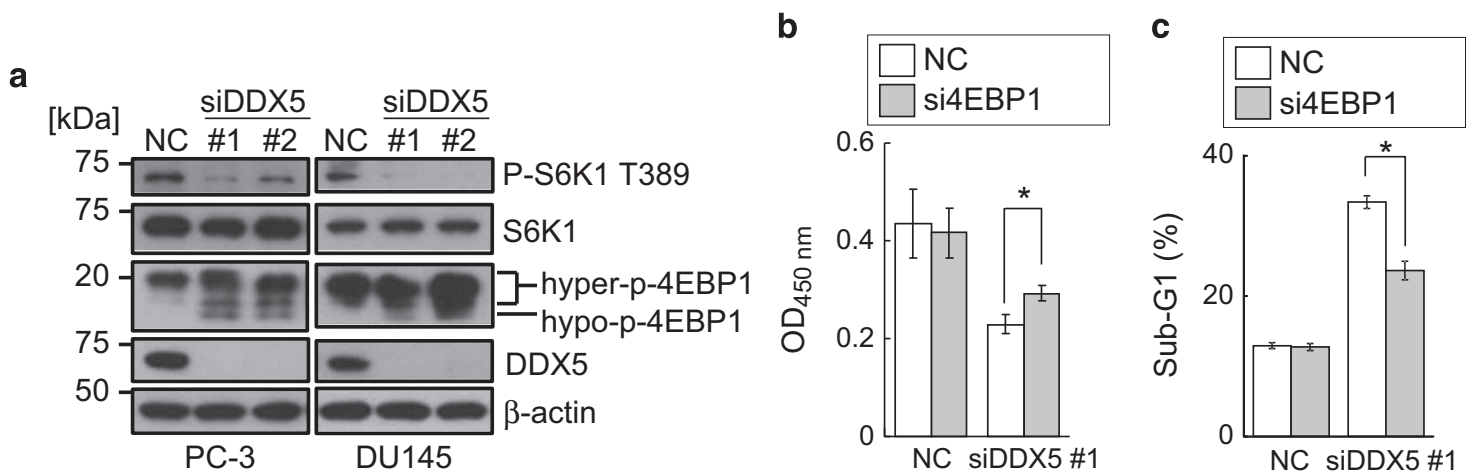

Figure 5 Knockdown of DDX5 inhibits the mTORC1 pathway of hormone-independent prostate cancer cells. (a) Western blotting analysis was performed after PC-3 and DU145 cells were transfected with or without siDDX5 and incubated for $72 \mathrm{~h}$. (b and c) After PC-3 cells were transfected with or without siDDX5 \#1 and/or si4EBP1 for $72 \mathrm{~h}$, CCK-8 assay (b) and quantification of apoptosis (c) were performed. Data are means \pm S.D. $(n=3)$. ${ }^{*} P<0.05$ relative to control (Student's $t$-test)
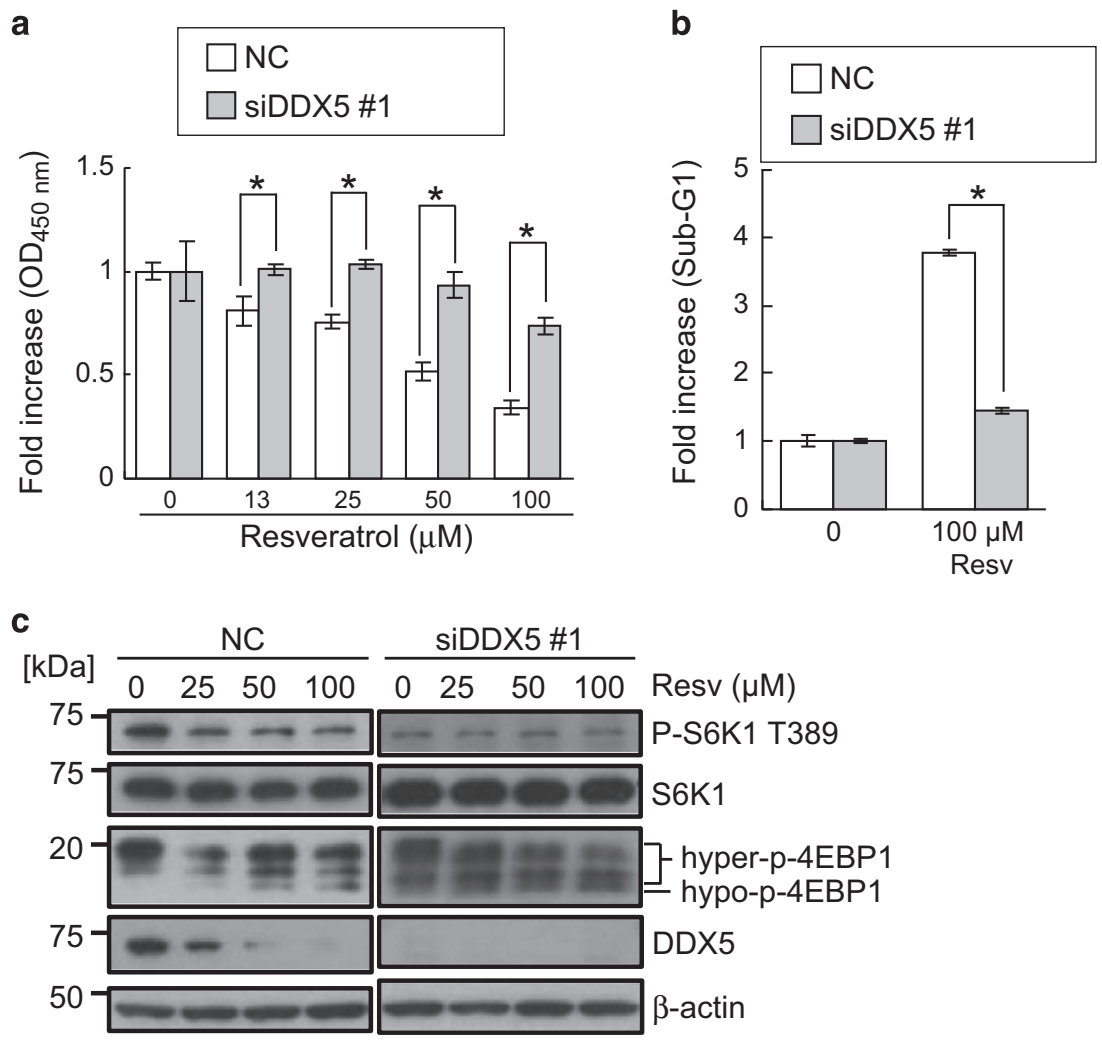

Figure 6 Resveratrol inhibits the mTORC1 pathway and growth of prostate cancer cells through depletion of DDX5. PC-3 cells were treated with resveratrol at the indicated concentrations for $24 \mathrm{~h}$ after transfected with a negative control siRNA (NC) or siDDX5 \#1 and incubated for $48 \mathrm{~h}$. (a) Relative viability was measured by CCK-8 assay. Data are means \pm S.D. $(n=3) .{ }^{*} P<0.05$ relative to control (Student's $t$-test). (b) Apoptosis was quantified by flow cytometric analysis. Data are means \pm S.D. $(n=3) .{ }^{*} P<0.05$ relative to control (Student's $t$-test). Resv; Resveratrol. (c) mTORC1 activity was examined by western blotting

may augment docetaxel efficacy against castration-resistant prostate cancer.

The present study suggested that resveratrol inhibits the mTORC1 pathway by targeting DDX5. Previous studies indicated that the suppression of MTORC1 signaling led not only to the inhibition of cancer progression ${ }^{22}$ but also to the amelioration of neurodegenerative disorders, ${ }^{48}$ alleviation of obesity-related diseases, ${ }^{49}$ and extension of lifespans. ${ }^{50}$
Therefore, resveratrol may exert these clinical benefits ${ }^{6,9,12}$ by targeting DDX5.

\section{Materials and Methods}

Reagents. trans-Resveratrol (Cat. No. 70675) and rolipram (Cat. No. 10011132) were purchased from Cayman Chemical (Ann Arbor, MI, USA). Purified recombinant proteins of Homo sapiens DEAD box helicase 5 (TP300371) and Homo sapiens phosphodiesterase 4A, cAMP-specific (TP307765) were purchased 


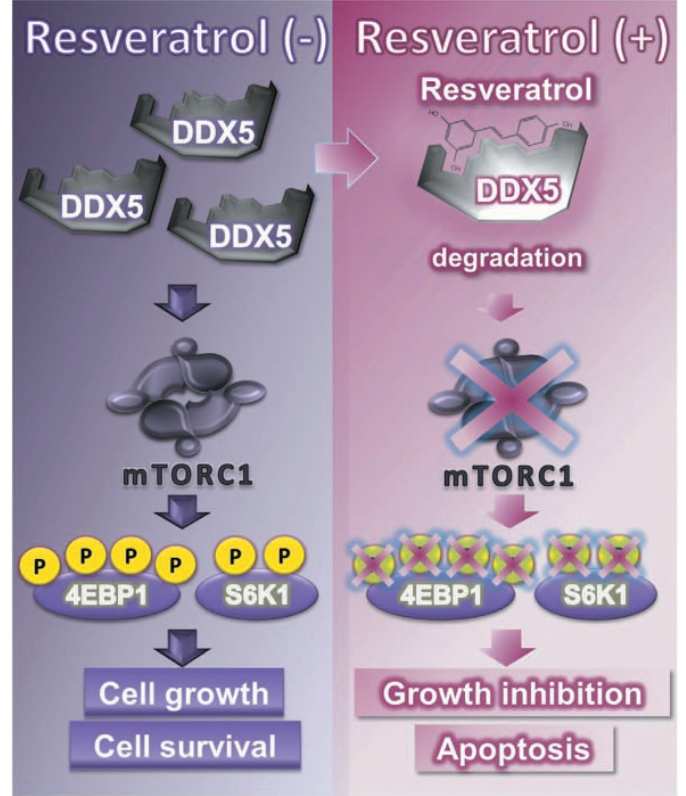

Figure 7 A schematic illustration of targeting the DDX5-mTORC1 axis by resveratrol. DDX5 protein is overexpressed in castration-resistant prostate cancer and promotes cell survival and growth by activating mTORC1 signaling. Resveratrol directly binds to DDX5 protein and promotes degradation of DDX5 protein, leading to suppression of the mTORC1 pathway, cancer cell survival, and growth

from OriGene Technologies (Rockville, MD, USA). Cycloheximide (Cat. No. 0674191) and pepstatin A (Cat. No. 26436-52) were purchased from Nacalai Tesque (Kyoto, Japan). Bafilomycin A1 (Cat. No. B-1080) was purchased from LC Laboratories (Woburn, MA, USA). Lactacystin (Cat. No. 4368-v), leupeptin (Cat. No. 4041), and antipain (Cat. No. 4062) were purchased from Peptide Institute (Osaka, Japan).

Cell culture. The human prostate cancer cell lines PC-3 and DU145 were obtained as the cell lines of $\mathrm{NCl}-60$ from the $\mathrm{NCl}$ Developmental Therapeutics Program. These cells were cultured in RPMl 1640 medium supplemented with $10 \%$ fetal bovine serum, $2 \mathrm{mM}$ L-glutamine, 50 units $/ \mathrm{ml}$ penicillin, and $100 \mu \mathrm{g} / \mathrm{ml}$ streptomycin at $37^{\circ} \mathrm{C}$ in $5 \% \mathrm{CO}_{2}$.

Cell viability assay. Cell viability was determined as previously described. ${ }^{38}$ Briefly, after the Cell Counting Kit-8 (CCK-8) solution (Dojindo Laboratories, Kumamoto, Japan) was added to the medium and incubated, the absorbance $(450 \mathrm{~nm})$ of the samples was measured.

Western blotting analysis. Western blotting analysis was performed as previously described. ${ }^{38}$ For detection of AMPK activation, PC-3 cells were fixed with ice-cold 10\% trichloroacetic acid in PBS on ice for 30 min. After scraping and washing with PBS, the cells were lysed with RIPA buffer and sonicated. The supernatants were subjected to SDS-PAGE and analyzed by western blotting. AntiDDX5 (PAb204, 05-850, Millipore, Billerica, MA, USA), 4EBP1 (\#9644, Cell Signaling Technology, Danvers, MA, USA), P-S6K T389 (\#9234, Cell Signaling Technology), S6K (\#2708, Cell Signaling Technology), P-ACC S79 (\#3661, Cell Signaling Technology), ACC (\#3676, Cell Signaling Technology), P-AMPK $\alpha$ T172 (\#2535, Cell Signaling Technology), AMPK $\alpha$ (\#2603, Cell Signaling Technology), PDE4 (ab14628, Abcam, Cambridge, UK), and $\beta$-actin (A5441, Sigma-Aldrich, St. Louis, MO, USA) antibodies were used.

Preparation of resveratrol-immobilized beads. Magnetic FG beads with epoxy linkers were purchased from Tamagawa Seiki (Nagano, Japan). The fixation of resveratrol onto the beads was performed as previously described. ${ }^{38}$ Briefly, the beads were incubated with $10 \mathrm{mM}$ trans-resveratrol in
$\mathrm{N}, \mathrm{N}$-dimethylformamide (DMF) containing potassium carbonate at $37^{\circ} \mathrm{C}$ for $24 \mathrm{~h}$ After being washed twice with DMF, the beads were then washed with Milli-Q water.

Purification and identification of resveratrol-binding proteins. Purification and identification of resveratrol-binding proteins were performed using resveratrol-fixed beads as previously described..$^{38}$ In the competitive binding assay, cell extracts were incubated with free trans-resveratrol for $1 \mathrm{~h}$ before incubation with the beads for $15 \mathrm{~min}$. Recombinant DDX5 protein was preincubated with or without $150 \mu \mathrm{g} / \mathrm{ml}$ RNase A (R4642, Sigma-Aldrich) for $30 \mathrm{~min}$ at room temperature and used for the binding assay.

Quantitative real-time RT-PCR. Quantitative RT-PCR analysis was performed as previously described.$^{51}$ Complementary DNA was amplified and quantified using TaqMan probes (Applied Biosystems, Foster City, CA, USA) to DDX5 (Hs01075383_g1), HES1 (Hs00172878_m1), kallikrein 4 (KLK4) (Hs00191772_m1), prostate carcinoma tumor antigen 1 (PCTA1) (Hs01057135_m1), TGM4 (Hs00162710_m1), PrLZ (Hs00893105_m1), and $\beta$-actin (Cat. No. 4352935E).

RNAi. The following siRNAs (Life Technologies, Carlsbad, CA, USA) were used: siDDX5 \#1 (HSS102717), 5'-GGUGCAGCAAGUAGCUGCUGAAUAU-3'; siDDX5 \#2 (HSS102715), 5'-GGAAUCUUGAUGAGCUGCCUAAAUU-3'; si4EBP1 (HSS141934), 5'-GGGUCACCAGCCCUUCCAGUGAUGA-3'; and a negative control siRNA (Cat. No. 12935-115). Only sense strands are shown. Transfection of siRNAs was performed as previously described. ${ }^{38}$

Colony-formation assay. PC-3 cells were seeded at a density of 200 cells/ well in six-well plates. The cells were transfected with each siRNA and incubated for 9 days. After being fixed with 10\% formalin, the colonies were stained with crystal violet. The total number of colonies was counted.

Quantification of apoptosis. Apoptosis was detected and quantified as previously described. ${ }^{51}$ Briefly, after washing with PBS, PC-3 cells were suspended in PBS containing $0.1 \%$ Triton X-100, $50 \mu \mathrm{g} / \mathrm{ml}$ propidium iodide, and $100 \mu \mathrm{g} / \mathrm{ml}$ RNase A. The percentage of hypodiploid DNA (sub-G1) was quantified as the ratio of apoptosis by FACSCalibur (Becton, Dickinson and Company, Franklin Lakes, NJ, USA).

Statistics. Data are represented as means and S.D. Statistical analyses were performed using one-way ANOVA followed by Bonferroni post-hoc tests or unpaired Student's $t$-test.

\section{Conflict of Interest}

The authors declare no conflict of interest.

Acknowledgements. This work was supported by JSPS Grant-in-Aids for Young Scientists (A) (No. 23689036) and JSPS Fellows (No. 13J10279).

1. Jang M, Cai L, Udeani GO, Slowing KV, Thomas CF, Beecher CW et al. Cancer chemopreventive activity of resveratrol, a natural product derived from grapes. Science 1997; 275: 218-220.

2. Baur JA, Sinclair DA. Therapeutic potential of resveratrol: the in vivo evidence. Nat Rev Drug Discov 2006; 5: 493-506.

3. Dolinsky VW, Chan AY, Robillard Frayne I, Light PE, Des Rosiers C, Dyck JR. Resveratrol prevents the prohypertrophic effects of oxidative stress on LKB1. Circulation 2009; 119: 1643-1652.

4. Renaud S, de Lorgeril M. Wine, alcohol, platelets, and the French paradox for coronary heart disease. Lancet 1992; 339: 1523-1526.

5. Kim D, Nguyen MD, Dobbin MM, Fischer A, Sananbenesi F, Rodgers JT et al. SIRT1 deacetylase protects against neurodegeneration in models for Alzheimer's disease and amyotrophic lateral sclerosis. EMBO J 2007; 26: 3169-3179.

6. Maher P, Dargusch R, Bodai L, Gerard PE, Purcell JM, Marsh JL. ERK activation by the polyphenols fisetin and resveratrol provides neuroprotection in multiple models of Huntington's disease. Hum Mol Genet 2011; 20: 261-270.

7. Baur JA, Pearson KJ, Price NL, Jamieson HA, Lerin C, Kalra A et al. Resveratrol improves health and survival of mice on a high-calorie diet. Nature 2006; 444: 337-342.

8. Lagouge M, Argmann C, Gerhart-Hines Z, Meziane H, Lerin C, Daussin F et al. Resveratro improves mitochondrial function and protects against metabolic disease by activating SIRT1 and PGC-1alpha. Cell 2006; 127: 1109-1122. 
9. Howitz KT, Bitterman KJ, Cohen HY, Lamming DW, Lavu S, Wood JG et al. Small molecule activators of sirtuins extend Saccharomyces cerevisiae lifespan. Nature 2003; 425: 191-196.

10. Patel KR, Brown VA, Jones DJ, Britton RG, Hemingway D, Miller AS et al. Clinical pharmacology of resveratrol and its metabolites in colorectal cancer patients. Cancer Res 2010; 70: 7392-7399

11. Smoliga JM, Baur JA, Hausenblas HA. Resveratrol and health - a comprehensive review of human clinical trials. Mol Nutr Food Res 2011; 55: 1129-1141.

12. Timmers S, Konings E, Bilet L, Houtkooper RH, van de Weijer T, Goossens GH et al. Calorie restriction-like effects of 30 days of resveratrol supplementation on energy metabolism and metabolic profile in obese humans. Cell Metab 2011; 14: 612-622.

13. Aggarwal BB, Bhardwaj A, Aggarwal RS, Seeram NP, Shishodia S, Takada Y. Role of resveratrol in prevention and therapy of cancer: preclinical and clinical studies. Anticancer Res 2004; 24: 2783-2840.

14. Lin SJ, Defossez PA, Guarente L. Requirement of NAD and SIR2 for life-span extension by calorie restriction in Saccharomyces cerevisiae. Science 2000; 289: 2126-2128.

15. Pacholec M, Bleasdale JE, Chrunyk B, Cunningham D, Flynn D, Garofalo RS et al. SRT1720, SRT2183, SRT1460, and resveratrol are not direct activators of SIRT1. J Biol Chem 2010; 285: 8340-8351.

16. Um JH, Park SJ, Kang H, Yang S, Foretz M, McBurney MW et al. AMP-activated protein kinase-deficient mice are resistant to the metabolic effects of resveratrol. Diabetes 2010; 59 : 554-563.

17. Park SJ, Ahmad F, Philp A, Baar K, Williams T, Luo H et al. Resveratrol ameliorates agingrelated metabolic phenotypes by inhibiting cAMP phosphodiesterases. Cell 2012; 148: 421-433.

18. Hubbard BP, Gomes AP, Dai H, Li J, Case AW, Considine T et al. Evidence for a common mechanism of SIRT1 regulation by allosteric activators. Science 2013; 339: 1216-1219.

19. Hopkins AL, Groom CR. The druggable genome. Nat Rev Drug Discov 2002; 1: 727-730.

20. Bishayee A. Cancer prevention and treatment with resveratrol: from rodent studies to clinical trials. Cancer Prev Res 2009; 2: 409-418.

21. Laplante M, Sabatini DM. mTOR signaling in growth control and disease. Cell 2012; 149 : 274-293.

22. Guertin DA, Sabatini DM. Defining the role of mTOR in cancer. Cancer Cell 2007; 12: 9-22.

23. Hsieh AC, Liu Y, Edlind MP, Ingolia NT, Janes MR, Sher A et al. The translational landscape of mTOR signalling steers cancer initiation and metastasis. Nature 2012; 485: 55-61.

24. Inoki K, Li Y, Zhu T, Wu J, Guan KL. TSC2 is phosphorylated and inhibited by Akt and suppresses mTOR signalling. Nat Cell Biol 2002; 4: 648-657.

25. Anjum R, Blenis J. The RSK family of kinases: emerging roles in cellular signalling. Nat Rev Mol Cell Biol 2008; 9: 747-758.

26. Gwinn DM, Shackelford DB, Egan DF, Mihaylova MM, Mery A, Vasquez DS et al. AMPK phosphorylation of raptor mediates a metabolic checkpoint. Mol Cell 2008; 30: 214-226.

27. Thedieck K, Holzwarth B, Prentzell MT, Boehlke C, Kläsener K, Ruf S et al. Inhibition of mTORC 1 by astrin and stress granules prevents apoptosis in cancer cells. Cell 2013; 154: 859-874.

28. Lukong KE, Chang KW, Khandjian EW, Richard S. RNA-binding proteins in human genetic disease. Trends Genet 2008; 24: 416-425.

29. van Kouwenhove M, Kedde M, Agami R. MicroRNA regulation by RNA-binding proteins and its implications for cancer. Nat Rev Cancer 2011; 11: 644-656.

30. Janknecht R. Multi-talented DEAD-box proteins and potential tumor promoters: p68 RNA helicase (DDX5) and its paralog, p72 RNA helicase (DDX17). Am J Transl Res 2010; 2. 223-234.

31. Mazurek A, Luo W, Krasnitz A, Hicks J, Powers RS, Stillman B. DDX5 regulates DNA replication and is required for cell proliferation in a subset of breast cancer cells. Cancer Discov 2012; 2: 812-825.

32. Han B, Mehra R, Dhanasekaran SM, Yu J, Menon A, Lonigro RJ et al. A fluorescence in situ hybridization screen for E26 transformation-specific aberrations: identification of DDX5ETV4 fusion protein in prostate cancer. Cancer Res 2008; 68: 7629-7637.

33. Saporita AJ, Chang HC, Winkeler CL, Apicelli AJ, Kladney RD, Wang J et al. RNA helicase DDX5 is a p53-independent target of ARF that participates in ribosome biogenesis. Cancer Res 2011; 71: 6708-6717.

34. Yang L, Lin C, Liu ZR. P68 RNA helicase mediates PDGF-induced epithelial mesenchymal transition by displacing Axin from beta-catenin. Cell 2006; 127: 139-155.
35. Cohen AA, Geva-Zatorsky N, Eden E, Frenkel-Morgenstern M, Issaeva I, Sigal A et al. Dynamic proteomics of individual cancer cells in response to a drug. Science 2008; 322 : 1511-1516.

36. Lin S, Tian L, Shen H, Gu Y, Li JL, Chen Z et al. DDX5 is a positive regulator of oncogenic NOTCH1 signaling in T cell acute lymphoblastic leukemia. Oncogene 2013; 32: 4845-4853.

37. Clark EL, Coulson A, Dalgliesh C, Rajan P, Nicol SM, Fleming S et al. The RNA helicase p68 is a novel androgen receptor coactivator involved in splicing and is overexpressed in prostate cancer. Cancer Res 2008; 68: 7938-7946.

38. lizumi $Y$, Oishi M, Taniguchi T, Goi W, Sowa $Y$, Sakai $T$. The flavonoid apigenin downregulates CDK1 by directly targeting ribosomal protein S9. PLoS One 2013; 8: e73219.

39. Li G, Rivas P, Bedolla R, Thapa D, Reddick RL, Ghosh R et al. Dietary resveratrol prevents development of high-grade prostatic intraepithelial neoplastic lesions: involvement of SIRT1/ S6K axis. Cancer Prev Res 2013; 6: 27-39.

40. Patel KR, Andreadi C, Britton RG, Horner-Glister E, Karmokar A, Sale $S$ et al. Sulfate metabolites provide an intracellular pool for resveratrol generation and induce autophagy with senescence. Sci Transl Med 2013; 5: 205ra133.

41. Hsieh AC, Costa M, Zollo O, Davis C, Feldman ME, Testa JR et al. Genetic dissection of the oncogenic mTOR pathway reveals druggable addiction to translational control via 4EBPelF4E. Cancer Cell 2010; 17: 249-261.

42. Jiang WG, Ye L, Sanders AJ, Ruge F, Kynaston HG, Ablin RJ et al. Prostate transglutaminase (TGase-4, TGaseP) enhances the adhesion of prostate cancer cells to extracellular matrix, the potential role of TGase-core domain. J Transl Med 2013; 11: 269.

43. Zhang D, He D, Xue Y, Wang R, Wu K, Xie H et al. PrLZ protects prostate cancer cells from apoptosis induced by androgen deprivation via the activation of Stat3/Bcl-2 pathway. Cancer Res 2011; 71: 2193-2202.

44. Williams S, Chiong E, Lojanapiwat B, Umbas R, Akaza H. Management of prostate cancer in Asia: resource-stratified guidelines from the Asian Oncology Summit 2013. Lancet Oncol 2013 14: e524-e534.

45. Yamaguchi T, Kakefuda R, Tajima N, Sowa Y, Sakai T. Antitumor activities of JTP-74057 (GSK1120212), a novel MEK1/2 inhibitor, on colorectal cancer cell lines in vitro and in vivo. Int J Oncol 2011; 39: 23-31.

46. Kai L, Levenson AS. Combination of resveratrol and antiandrogen flutamide has synergistic effect on androgen receptor inhibition in prostate cancer cells. Anticancer Res 2011; 31: 3323-3330.

47. Morikawa $Y$, Koike $H$, Sekine $Y$, Matsui $H$, Shibata $Y$, Ito $K$ et al. Rapamycin enhances docetaxel-induced cytotoxicity in a androgen-independent prostate cancer xenograft model by survivin downregulation. Biochem Biophys Res Commun 2012; 419: 584-589.

48. Ravikumar B, Vacher C, Berger Z, Davies JE, Luo S, Oroz LG et al. Inhibition of mTOR induces autophagy and reduces toxicity of polyglutamine expansions in fly and mouse models of Huntington disease. Nat Genet 2004; 36: 585-595.

49. Le Bacquer O, Petroulakis E, Paglialunga S, Poulin F, Richard D, Cianflone K et al. Elevated sensitivity to diet-induced obesity and insulin resistance in mice lacking 4E-BP1 and 4E-BP2. $J$ Clin Invest 2007; 117: 387-396.

50. Harrison DE, Strong R, Sharp ZD, Nelson JF, Astle CM, Flurkey K et al. Rapamycin fed late in life extends lifespan in genetically heterogeneous mice. Nature 2009; 460: 392-395.

51. Oishi M, lizumi Y, Taniguchi T, Goi W, Miki T, Sakai T. Apigenin sensitizes prostate cancer cells to Apo2L/TRAIL by targeting adenine nucleotide translocase-2. PLoS One 2013; 8: e55922.

(i) Cell Death and Disease is an open-access journal published by Nature Publishing Group. This work is licensed under a Creative Commons Attribution 4.0 International License. The images or other third party material in this article are included in the article's Creative Commons license, unless indicated otherwise in the credit line; if the material is not included under the Creative Commons license, users will need to obtain permission from the license holder to reproduce the material. To view a copy of this license, visit http://creativecommons.org/licenses/by/4.0/ 